\title{
Strain-specific interaction of Fructilactobacillus sanfranciscensis with yeasts in the sourdough fermentation
}

\author{
Esther Rogalski ${ }^{1} \cdot$ Matthias A. Ehrmann ${ }^{1} \cdot$ Rudi F. Vogel ${ }^{1}$
}

Received: 23 January 2021 / Revised: 10 March 2021 / Accepted: 12 March 2021 / Published online: 22 April 2021

(c) The Author(s) 2021

\begin{abstract}
Fructilactobacillus $(F$.) sanfranciscensis is a key bacterium in traditional (type 1) sourdough fermentations. It typically occurs in combination with the sourdough yeast Kazachstania (K.) humilis or the generalist Saccharomyces (S.) cerevisiae. Previous studies revealed intra-species diversity in competitiveness or dominance in sourdoughs of $F$. sanfranciscensis, as well as preferences for a life with or without a specific yeast. In this study representative, differently behaving strains were studied in media with different sugars and electron acceptors, and in rye sourdough fermentations in the presence and absence of $K$. humilis or S. cerevisiae. Strain-specific differences were observed in sugar and organic acids spectra in media, and in sourdoughs with $F$. sanfranciscensis strains in combination with $K$. humilis or S. cerevisiae. F. sanfranciscensis TMW 1.1150 proved dominant in the presence and absence of any yeast because it most effectively used maltose. Its maltose fermentation was unaffected by electron acceptors. F. sanfranciscensis TMW 1.2138 was the weakest maltose fermenter and incapable of glucose fermentation, and evidently not competitive against the other strains. F. sanfranciscensis TMW 1.392 was the most versatile strain regarding the utilization of different carbohydrates and its ability to exploit electron acceptors like fructose and oxygen. In sourdoughs without yeasts, it outcompeted other strains. The metabolism of $F$. sanfranciscensis TMW 1.907 was stimulated in combination with S. cerevisiae. In competitive trials, it was assertive only with S. cerevisiae. The intra-species differences in carbohydrate metabolism can widely explain the differences in their behavior in sourdough fermentation. Interaction between $F$. sanfranciscensis and the yeasts was strain specific and supposedly commensal with $K$. humilis and rather competitive with S. cerevisiae.
\end{abstract}

Keywords Kazachstania humilis · Saccharomyces cerevisiae $\cdot$ Interaction · Electron acceptors · Sourdough fermentation · Competition $\cdot$ Carbohydrates $\cdot$ HPLC analysis

\section{Introduction}

The heterofermentative lactic acid bacterium (LAB) Fructilactobacillus $(F$.) sanfranciscensis (formerly Lactobacillus sanfranciscensis) is a key species in traditional type1-sourdough fermentations [1-4]. These fermentations last between 4 and $16 \mathrm{~h}$ and take place at medium temperatures and a $\mathrm{pH}$ between 3.7 and 4 [5, 6]. The fermentation conditions fit perfectly to the growth requirements of $F$. sanfranciscensis [7]. During fermentation, $F$. sanfranciscensis produces lactate, acetate, ethanol and carbon dioxide [8, 9].

Rudi F. Vogel

rudi.vogel@tum.de

1 Lehrstuhl Für Technische Mikrobiologie, Technische Universität München, Gregor-Mendel-Straße 4, 85354 Freising, Germany
The yeasts Kazachstania (K.) humilis (previously named Candida humilis) and Saccharomyces (S.) cerevisiae are also common inhabitants of sourdough [10,11]. Whereas $K$. humilis is a typical sourdough yeast, which is only found in this niche, $S$. cerevisiae is a generalist with many biotypes $[1,12]$.

The microorganisms in the niche sourdough need to combat a stressful ecosystem. It is characterized by specialized offer of high and low molecular substrates and electron acceptors as well as high acidity and redox stress. Moreover, an adaption to the carbohydrates and nutritional options is required [2, 12-14]. F. sanfranciscensis is perfectly adapted to the sourdough surrounding. Maltose is its preferred carbohydrate, which is together with glucose constantly produced by the flour amylases from starch [15, 16]. Still, there are strain-specific differences in the utilization of carbohydrates between the $F$. sanfranciscensis strains 
$[8,17]$. Sucrose, which is also present in the dough, and fructose (derived thereof) can be utilized in the metabolism by specific $F$. sanfranciscensis strains [8, 18-20]. Most of the $F$. sanfranciscensis strains are able to use fructose as an external electron acceptor for the recycling of NAD [4, 21]. Fructose is available in the sourdough due to the cleavage of glucofructans by specific yeasts like $K$. humilis and $S$. cerevisiae [22]. The yeasts, particularly $K$. humilis as maltose-negative yeast, use the resulting glucose for their metabolism [12, 23]. Consequently, there is no competition for the maltose in the sourdough between these two yeasts. As glucose can be released from the maltose phosphorylase reaction when maltose is abundant by $F$. sanfranciscensis, an often-found combination is $F$. sanfranciscensis and the yeast K. humilis, e.g. for strain TMW 1.392 (LTH 2590) [7, 21, $24,25]$. It, therefore, has been reasoned that this combination, which is often found in rye sourdough fermentations, is based on mutualism or may also result from indirect interactions based on glutathione and other thiol-metabolism, which act on the redox potential [1, 14, 23]. S. cerevisiae is often found in the bakeries surrounding and it is, therefore, assumed that it is also found in the sourdough [26, 27]. This yeast is a generalist as it can utilize maltose and glucose and various other sugars like sucrose as carbohydrate source [28]. Still, the $S$. cerevisiae sourdough isolates are acid resistant, which is not necessarily the case for the strains used for dough leavening [12, 29]. Otherwise, due to the usage of maltose by S. cerevisiae, F. sanfranciscensis is in general nutrient competition with the yeast. This stress is demonstrated by an increase of the maltose phosphorylase, which cleaves maltose in glucose-1-phosphate and glucose [21]. The glucose-1-phosphate is utilized in the metabolism of $F$. sanfranciscensis whereas the glucose is secreted in the abundance of maltose. The massive segregation of glucose leads to the glucose repression in many other LABs as well as in $S$. cerevisiae [28, 30]. This effect supposedly detains the $S$. cerevisiae from the uptake of maltose during the sourdough fermentation by glucose repression. In competition studies, it was found that the cell count of yeasts, especially $S$. cerevisiae and $K$. humilis, is always higher in the dough in the absence of $\operatorname{LAB}[23,29]$. This result illustrates a competitive influence of the LAB on the yeast. Furthermore, an intra-species competition between $F$. sanfranciscensis strains exists. It is possible that more than one strain of $F$. sanfranciscensis is present in a sourdough fermentation [31]. This phenomenon can be the result of a selection for strains in a distinct fermentation based on an intra-species competition or due to a coincidence by contamination form different sourdoughs. Moreover, in competition studies, a clear competition between strains in one sourdough fermentation was demonstrated [23, 32]. The strain-specific competition in the sourdough was independent or dependent on the yeast inoculated in the sourdough fermentation [23]. A genotype-phenotype study of $F$. sanfranciscensis showed that these strains have several differences in their carbohydrate utilization and their use of external electron acceptors [8, 17]. The present study was, therefore, dedicated to elucidate mechanisms of the strain-specific interaction between $F$. sanfranciscensis and yeasts in the sourdough fermentation by comparison of carbohydrate metabolism with their behavior in combination with yeasts in rye sourdough fermentations, and in previous competition studies.

\section{Materials and methods}

\section{Strains and culture conditions}

The $F$. sanfranciscensis strains TMW 1.1150, TMW 1.392, TMW 1.907 and TMW 1.2138 as well as the yeasts K. humilis TMW 3.1034 and S. cerevisiae TMW 3.1064 were chosen from the TMW strain collection based on their (different) competitiveness against other strains in the sourdough system and their genomic diversity $[8,23]$. In the TMW strain collection, different yeast and lactic acid bacteria of different food fermentations were collected and stored. The strains were grown at $30^{\circ} \mathrm{C}$ for $48 \mathrm{~h}$ in static conditions in modified DeMan Rogosa and Sharpe media (mMRS) [32]. The yeasts K. humilis TMW 3.1034 and S. cerevisiae TMW 3.1064 were grown overnight in yeast peptone glucose (YPG) media at $30{ }^{\circ} \mathrm{C}$. For agar plates, $15 \%$ AgarAgar (Roth, Karlsruhe, Germany) was added to the media. For glycerol stocks, the overnight cultures were centrifuged, and the cell pellet was mixed with $70 \%$ glycerol and stored at $-80{ }^{\circ} \mathrm{C}$.

\section{Sourdough and sample preparation}

Overnight cultures of the $F$. sanfranciscensis strains TMW 1.392, TMW 1.907, TMW 1.1150 and TMW 1.2138 were adjusted to an $\mathrm{OD}_{600}$ of 5 in $14 \mathrm{ml} 1 / 4$ Ringer's solution and 
were added to $100 \mathrm{~g}$ whole meal rye flour (dm, Karlsruhe, Germany) and $86 \mathrm{~g}$ tap water. For each strain, three separate sourdoughs were prepared, one without any yeast (- yeast) and one with the yeasts $K$. humilis TMW 3.1034 or S. cerevisiae TMW 3.1064. The yeast was added in a ratio of 1:100 to the bacterial cell count to the pre-fermented sourdough mixture simultaneously with the $F$. sanfranciscensis strain. The sourdough was propagated with $5 \%$ to the flour mass with a dough yield of 200 . After three times of sourdough propagation, the sourdough was back slopped again for propagation and samples were taken after 0 and $24 \mathrm{~h}$ of the fermentation for DNA isolation, colony-forming units $(\mathrm{cfu}) / \mathrm{ml}$ and high-throughput analysis matrix-assisted laser desorption/ionization (MALDI) time of flight (ToF) mass spectrometry (MS) measurements and HPLC analysis. During the whole fermentation process, the $\mathrm{pH}$ was measured before and after propagation. Furthermore, DNA isolation and the CLLP-PCR for strain identification were performed according to Rogalski et al. [32]. For the determination of the colony-forming units, $10 \mathrm{~g}$ of sourdough was mixed with $90 \mathrm{ml}$ of $1 / 4$ Ringer`s solution (Merck, Darmstadt, Germany) and a tenfold serial dilution up to $10^{-7}$ was performed. Furthermore, the dilution steps were plated out on mMRS and YPG agar plates and incubated for $48 \mathrm{~h}$ at $30^{\circ} \mathrm{C}$ aerobically. The colonies were counted and 48 of each plate were applied for MALDI ToF MS analysis (MS, Bruker, Billerica, USA).

\section{Analytical analysis of carbohydrates and organic acids}

Overnight cultures of the $F$. sanfranciscensis strains were prepared anaerobically in mMRS media under static conditions. The cultures were centrifuged at $7000 \mathrm{~g}$ for $7 \mathrm{~min}$, washed with $1 / 4$ Ringer's solution and adjusted to an $\mathrm{OD}_{600}$ of 5. Afterwards, the concentration was adjusted to $20 \mathrm{mM}$ for maltose (GEBRU Biotechnik GmbH, Heidelberg, Germany), glucose (Merck), fructose (Omni Life Science GmbH \& Co. KG, Bremen, Germany), sucrose (GEBRU Biotechnik) or ribose (Roth). To test the response of the strains to external electron acceptors, the combination of $20 \mathrm{mM}$ maltose with $20 \mathrm{mM}$ fructose, citrate (Roth), Na-gluconate (Roth) or malate (Sigma-Aldrich) was added to the cultures. To test the reaction with oxygen, cultures with $20 \mathrm{mM}$ maltose were incubated in Erlenmeyer flasks at $150 \mathrm{rpm}$. The rest of the cultures was incubated at static conditions at $30{ }^{\circ} \mathrm{C}$ for $6 \mathrm{~h}$. Samples were taken after $0 \mathrm{~h}$ and $6 \mathrm{~h}$. Subsequently, the cultures were centrifuged for $10 \mathrm{~min}$ at $14,000 \mathrm{xg}$ and the supernatant was filtered two times and added to HPLC vials for organic acid determinations (Phenomenex, Torrance, USA).

Also, sourdough samples were prepared for the HPLC analysis. Therefore, the sourdough samples were mixed 1:2 $\mathrm{w} / \mathrm{v}$ in deionized water and centrifuged at $8000 \times g, 10{ }^{\circ} \mathrm{C}$ for $30 \mathrm{~min}$. For the analysis of organic acids and ethanol, 5\% perchloric acid (70\%) was added and incubated overnight at $4{ }^{\circ} \mathrm{C}$ [33]. Afterwards, the supernatant was filtered with $2 \mu \mathrm{M}$ membrane filters (Phenomenex, Germany). A different sample preparation was used for the analysis of carbohydrates. Here the centrifuged supernatant was incubated with $12.52 \mathrm{mM} \mathrm{ZnSO}{ }_{4} \cdot 7 \mathrm{H}_{2} \mathrm{O}$ (Carrez solution 2), $10 \mathrm{mM}$ $\mathrm{NaOH}$ and $4.26 \mathrm{mM} \mathrm{K} \mathrm{K}_{4}\left[\mathrm{Fe}\left(\mathrm{CN}_{6}\right)\right] \cdot 3 \mathrm{H}_{2} \mathrm{O}$ (Carrez solution $1)$, centrifuged and also sterile filtered [33, 34].

Subsequently, for the analysis of organic acids and alcohols, a sulfonated styrene-divinylbenzene Rezex ROA column (Phenomenex), with $0.005 \mathrm{~N} \mathrm{H}_{2} \mathrm{SO}_{4}$ as mobile phase, and for the analysis of sugars and sugar alcohols, a Rezex RPM column (Phenomenex) with deionized water as mobile phase were applied at $85^{\circ} \mathrm{C}$. Furthermore, an injection volume of $20 \mu \mathrm{l}$ with a flow rate of $0.6 \mathrm{ml} / \mathrm{min}$ was chosen. The columns were coupled to a refractive index detection (RI) (ERC Refractomax 521, Thermo Fisher Scientific). The acids, sugars and sugar alcohols were identified and qualified with standards and the data were analyzed with Chromeleon ${ }^{\mathrm{TM}}$ software (Version 6.8, Dionex, Germany) [35]. Afterwards, the fermentation quotient (FQ) was calculated as the ratio of lactate to acetate for the sourdough samples as well as the ratio between lactate and ethanol [33]. The turnover of the metabolites during the $6 \mathrm{~h}$ of incubation was calculated. For the depletion of the substances, a ratio between the values of $6 \mathrm{~h}$ to $0 \mathrm{~h}$ of incubation time was calculated per g/cell dry mass. Furthermore, the production of the substances was calculated between the values of $0 \mathrm{~h}$ to $6 \mathrm{~h}$ of incubation time per $\mathrm{g} / \mathrm{cell}$ dry mass. The uncalculated values were provided in the Figs. A1, 2, 3, 4 together with the standard deviation.

\section{Determination of the cellular dry weight, morphology, and cell size}

The cfu $/ \mathrm{ml}$ and cellular dry weight of the $F$. sanfranciscensis strains were determined at an $\mathrm{OD}_{600}$ of 5 . Therefore, overnight cultures were grown and set to an $\mathrm{OD}_{600}$ of 5 with $1 / 4$ Ringer`s solution. The determination of the cell count was performed as mentioned above. For the determination of the cellular dry weight, falcons were set in a desiccator for $1 \mathrm{~h}$ and weighed. The cultures with an $\mathrm{OD}_{600}$ of 5 were added to the pre-weighted falcons and centrifuged at $10,000 \times g$ for $10 \mathrm{~min}$ at RT. The cell pellet was dried for $24 \mathrm{~h}$ at $95^{\circ} \mathrm{C}$, cooled down for $1 \mathrm{~h}$ in a desiccator to RT and the falcon with the cell pellet was weighed again. For the measurements of the cell size, cells out of an overnight culture were examined under a light microscope (Axiostar Plus, Carl Zeiss AG, Oberkochen, Germany) and the cell size was determined with a $5 \mu \mathrm{m}$ standard of the ZEN Blue Edition software (Carl Zeiss AG). 


\section{Statistical analysis}

All experiments were performed in biological triplicates. In case of the determination of the cell count and the MALDI ToF MS analysis, technical duplicates were performed $(n=6)$. For analysis of the cell size, a twosided Student's $t$ test was applied. Furthermore, a one-way ANOVA was applied to analyze the metabolic differences in the sourdough when yeasts or no yeasts were inoculated. Therefore, only bacterial products like lactate, acetate and mannitol were calculated; results $p<0.05$ were considered significant. The standard deviation was calculated for all analytical results. Outliers broader than $10 \%$ percent were ignored.

\section{Results}

\section{Differences in cell morphology of $F$. sanfranciscensis strains}

There are strain-specific differences in the cell morphology, cell size and cell weight of $F$. sanfranciscensis (Table 1; Fig. 1), which need to be considered in the comparison of metabolic turnover. TMW 1.392 and TMW 1.1150 have shorter/smaller cells than TMW 1.907 and TMW 1.2138. In the latter case, these two strains have also a broader variety in their cell morphology. The median cell size is about $5 \mu \mathrm{m}$ with a large distribution in their cell size. Considering TMW 1.392 and TMW 1.1150, the cell size differs only slightly between the single cells (Fig. 1a, c). Furthermore, the cell sizes between the strains differed significantly from each other, with the exception of $F$. sanfranciscensis TMW 1.907 to TMW 1.2138 (Fig. 1). The differences in the cell size reflect the number of cells found in a solution of an $\mathrm{OD}_{600}$ of 5. The smaller the single cell, the higher the cell count of the strain in a defined solution (Table 1).
For $F$. sanfranciscensis strains TMW 1.907, TMW 1.2138 and TMW 1.1150, the measured cell size fits to the resulting $\mathrm{cfu} / \mathrm{ml}$ and the cell dry weight at an $\mathrm{OD}_{600}$ of 5. F. sanfranciscensis TMW 1.907 and TMW 1.2138 had a high cell dry weight with a low cell count with larger cells. In addition, the $F$. sanfranciscensis strain TMW 1.1150 had the highest cell count, however, only at a medium cell dry weight because of its small cells. The cell dry weight differs from the cell size and the resulting cell count in $F$. sanfranciscensis TMW 1.392. F. sanfranciscensis TMW $1.392 \mathrm{had}$ a cfu/ $\mathrm{ml}$ of $6.16 \times 10^{9}$ in a culture with an $\mathrm{OD}_{600}$ of 5 although it had the lowest cell dry weight (Table 1).

\section{The turnover of carbohydrates is strain dependent}

The $F$. sanfranciscensis strains differ in their competitiveness in the sourdough and their genetic equipment [8, 23]. This should be reflected in the metabolism. Different sugars were chosen, which are common in sourdough fermentation, and the turnover is given in relation to the cell dry weight (Table 1; Fig. 2). Fermentation of a carbohydrate was recorded only when metabolites like lactate, acetate or ethanol were produced. Maltose was fermented by all strains within $6 \mathrm{~h}$ of incubation. F. sanfranciscensis TMW 1.1150 was the strongest maltose fermenter followed by TMW 1.392 (Fig. 2a). In the glucose fermentation, $F$. sanfranciscensis TMW 1.1150 showed the strongest turnover after $6 \mathrm{~h} \mathrm{com-}$ pared to the other strains. In TMW 1.907 and TMW 1.392 a glucose turnover was recorded resulting in the production of lactate and ethanol, and very low amounts of acetate. Fructose and sucrose were degraded only by $F$. sanfranciscensis TMW 1.392. When fructose is degraded, lactate, mannitol, and acetate instead of ethanol was produced. The same turnover can be seen in the degradation of sucrose by F. sanfranciscensis TMW 1.392 (Fig. 2). There is no degradation of ribose. Only in F. sanfranciscensis TMW 1.1150, a turnover of ribose appears possible as with the degradation
Fig. 1 Light electron microscopy image of $F$. sanfranciscensis strain a TMW 1.392, b TMW 1.907, c TMW 1.1150 and d TMW 1.2138. Size bars correspond to $5 \mu \mathrm{m}$, recordings are performed with ZEN Blue image software. The cell size in $\mu \mathrm{m}$ is illustrated in e) with the median and standard deviation. Bars with a different lowercase letter are differing statistically $(p<0.05)$ from each other

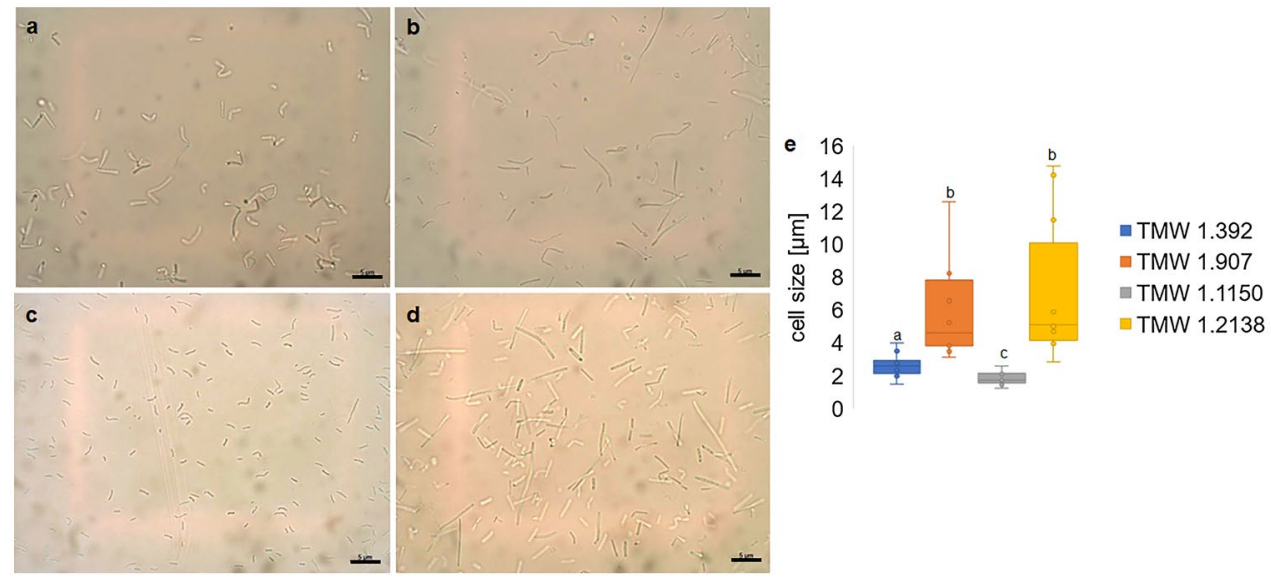



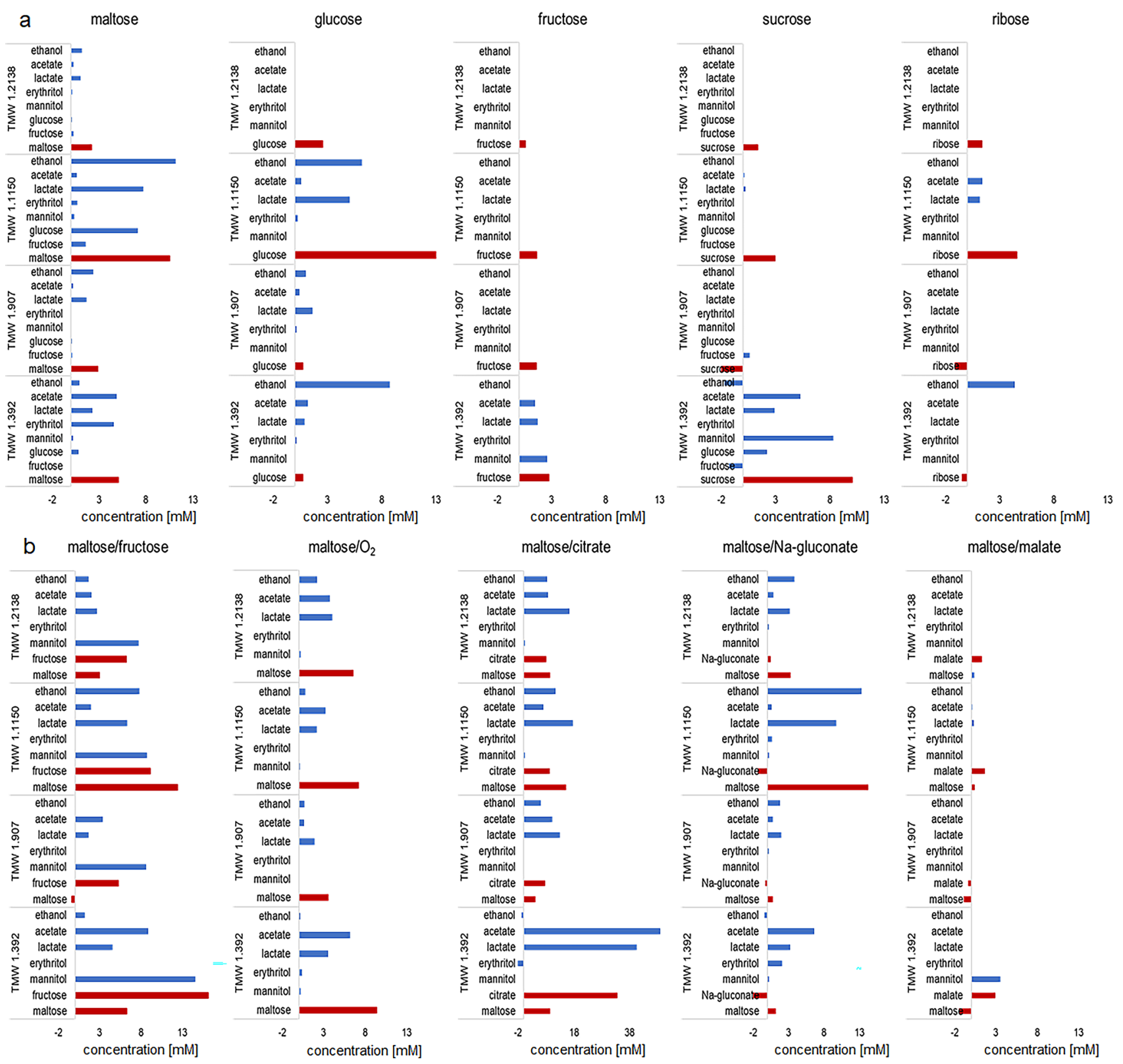

Fig. 2 Turnover of sugars and electron acceptors of $F$. sanfranciscensis TMW 1.392, TMW 1.907, TMW 1.1150, TMW 1.2138 in relation to the dry mass determined with HPLC analysis. The red bar indicates the consumption of the carbohydrate and the blue bar represents the production of the products during $6 \mathrm{~h}$ of incubation in Ringer's solution with $20 \mathrm{mM}$ of each reagent. In a the fermentation with one sugar was presented and in $\mathbf{b}$ the fermentation with maltose and an additional electron acceptor was pictured of ribose a production of acetate and lactate occurs (Fig. 2). In the fermentation of maltose and glucose, erythritol is produced every time in combination with acetate.

The enhancement of the maltose uptake in combination with fructose, oxygen, citrate, Na-gluconate and malate were determined (Fig. 2b). All strains produced more mannitol and less ethanol in combination with fructose, than solely with maltose (Fig. 2b). Moreover, no erythritol is produced in combination with external electron acceptors. When malate is added in combination with maltose, the maltose 
Fig. 3 Development of the $\mathrm{pH}$ values (a) and the cell count (b) of the sourdoughs between 0 and $24 \mathrm{~h}$ of fermentation. The sourdoughs were investigated from the $F$. sanfranciscensis strains TMW 1.392, TMW 1.907, TMW 1.1150 and TMW 1.2138 in combination without yeasts (-yeast), with $K$. humilis TMW $3.1034(+K$. humilis $)$ or with $S$. cerevisiae TMW 3.1064 $(+S$. cerevisiae $)$ respectively

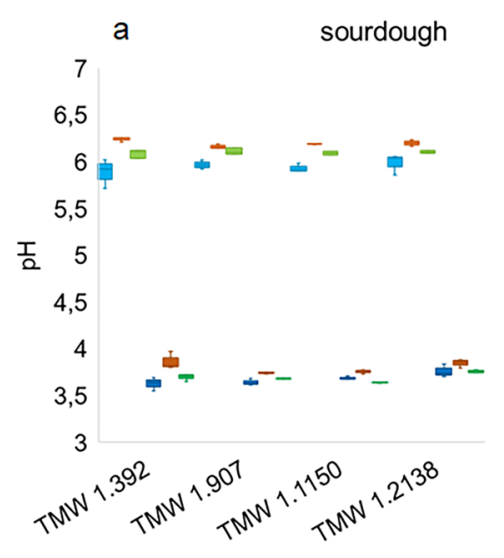

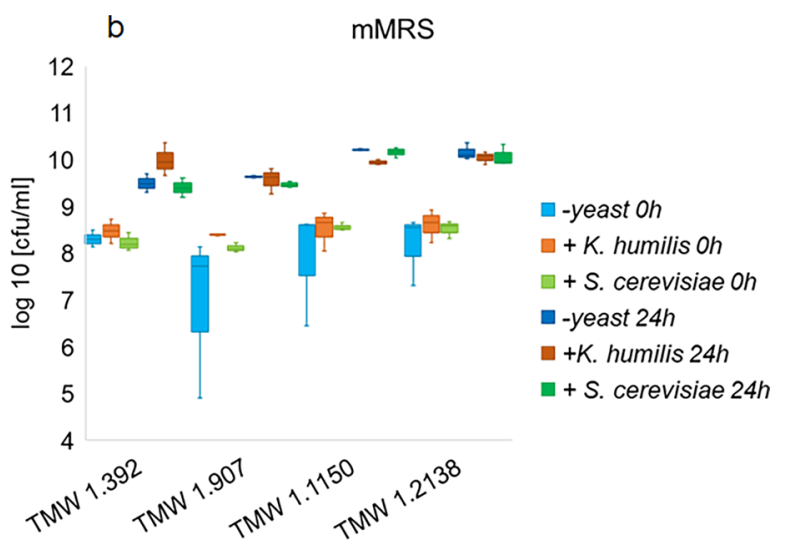

uptake and turnover are decreased in all strains. The metabolism of $F$. sanfranciscensis TMW 1.392 was increased the most compared to the other strains by the addition of external electron acceptors like fructose, oxygen, and citrate. In combination with citrate, the production of lactate and especially acetate is increased the most, although the depletion of maltose is not increased (Fig. 2b). During the turnover of maltose in combination with Na-gluconate, mostly more lactate and ethanol were produced. Similar to the reaction with only maltose, erythritol and acetate were produced when $\mathrm{Na-gluconate}$ is added to the reaction.

\section{The presence of yeasts influences the metabolic turnover in a sourdough fermentation by $F$. sanfranciscensis}

All four $F$. sanfranciscensis strains were evaluated with regard to their metabolic performance in a rye sourdough fermentation in response to the presence of yeasts. For this purpose, the single strains were added together with no yeast (-yeast), K. humilis 3.1034 or S. cerevisiae 3.1064 in sourdough fermentation. No yeast growth was recorded in the samples without any added yeasts. The development of the $\mathrm{pH}$ was comparable in sourdoughs with the different strains alone. Still, the sourdough without any yeasts and the sourdough with $S$. cerevisiae 3.1064 was slightly more acidic than the sourdough with K. humilis 3.1034 (Fig. 3a). The development of the cfu/ml between 0 and $24 \mathrm{~h}$ was similar for the strains. It increases from around 8 to $10 \log 10$ [cfu/ $\mathrm{ml}$ ] within $24 \mathrm{~h}$. In all -yeast fermentations a broad cfu/ $\mathrm{ml}$ standard deviation at $0 \mathrm{~h}$ was observed, except for the sourdough with $F$. sanfranciscensis TMW 1.392 (Fig. 3b). In Fig. 4 , the metabolites determined at $0 \mathrm{~h}$ and $24 \mathrm{~h}$ of fermentation of each strain and yeast combination are depicted. The same metabolites were detected with and without yeasts
(Fig. 4). The amount of ethanol was higher in the presence of yeasts because of their alcoholic fermentation. Furthermore, in the presence of S. cerevisiae TMW 3.1064 and in combination with $F$. sanfranciscensis TMW 1.392 and TMW 1.2138 the ethanol concentration was significantly the highest. In the absence of yeast, the amount of ethanol was very similar and in combination with $F$. sanfranciscensis TMW 1.392 greater than with the other strains. Apart from the ethanol concentration, there were no significant differences between the sourdoughs with the two yeasts. It should be noted that it is not possible to measure maltose during the fermentation as it is depleted directly after its production (by amylases) and is, therefore, below the detection limit. Small amounts of glucose were determined during the fermentation. The highest amount of glucose can be measured in the fermentation -yeast with F. sanfranciscensis TMW 1.2138 (Fig. 4c).

The FQ as well as the ratio between lactate and ethanol are major factors to evaluate a sourdough fermentation as it delivers a main sensory characteristic (Table 2). A fermentation with a low FQ has a high amount of acetate compared to lactate. In most of the strains, except $F$. sanfranciscensis TMW 1.2138 , the FQ is higher without any yeasts, which is the result of a low acetate concentration. In the $F$. sanfranciscensis strains TMW 1.1150, TMW 1.392 and TMW 1.907, the sourdough in combination with $S$. cerevisiae 3.1064 had the lowest FQ. The lowest FQ of all fermentations can be measured for the combination of $F$. sanfranciscensis TMW 1.2138 and $S$. cerevisiae TMW 3.1064 (Table 2). The ratio between lactate and ethanol was always higher in the absence of yeasts (lower amount of ethanol) and lower in the presence of yeasts. This observation is in line with the results above and the alcoholic fermentation of the yeasts (Fig. 3). In TMW 1.1150 and TMW 1.907 , the differences in the lactate ethanol ratio were minor in the fermentation without yeasts and with $K$. humilis TMW 
Fig. 4 HPLC analysis of the sourdough fermentation after 3 times of propagation at $0 \mathrm{~h}$ and $24 \mathrm{~h}$. The $F$. sanfranciscensis strains TMW 1.392, TMW 1.907, TMW 1.1150 and TMW 1.2138 in combination without yeast (a) with $K$. humilis TMW 3.1034 (b) or S. cerevisiae TMW 3.1064 (c). Bars with a different lowercase letter are differing statistically $(p<0.05)$ from each other
70

60

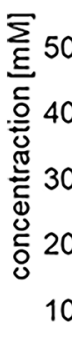

60

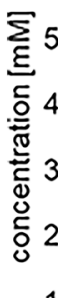

Oh

TMW 1.392

b
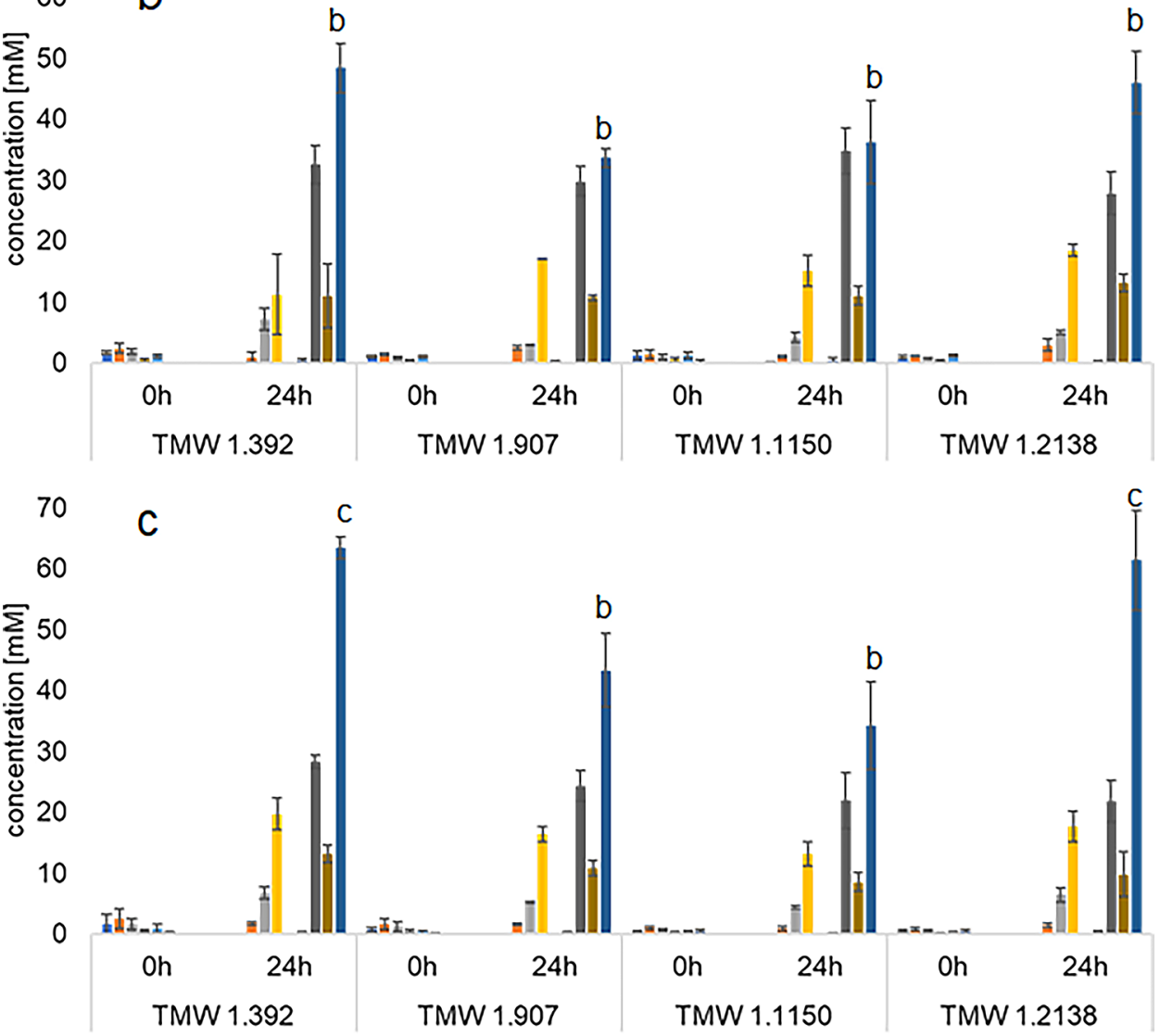

$\square$ maltose $\square$ glucose $\square$ fructose $\|$ mannitol $\square$ sucrose $\square$ citrate $\square$ lactate $\square$ acetate $\square$ ethanol
3.1034 (Table 2). A one-way ANOVA analysis was applied to evaluate the statistical differences between the inoculation with and without yeasts (-yeast) for all $F$. sanfranciscensis strains. There were no differences between the inoculation of yeasts in the production of lactate and acetate in the $F$. sanfranciscensis strains TMW 1.2138 and in case of TMW 1.392 in the production of lactate and mannitol. Furthermore, the difference in acetate production was significant $(p<0.05)$ between the fermentation of $K$. humilis TMW 3.1034 and $S$. cerevisiae TMW 3.1064 of $F$. sanfranciscensis TMW 1.392, TMW 1.907 and TMW 1.1150. In F. sanfranciscensis TMW 1.1150, the acetate production in all yeast combinations was significantly 
Table 1 Cell count (cfu/ml) and cell dry weight of the F. sanfranciscensis strains TMW 1.392, TMW 1.907, TMW 1.1150, TMW 1.2138 at an $\mathrm{OD}_{600}$ of 5

\begin{tabular}{lll}
\hline F. sanfranciscensis strain & $\mathrm{cfu} / \mathrm{ml}$ & $\begin{array}{l}\text { Cell dry } \\
\text { weight } \\
(\mathrm{mg} / 50 \mathrm{ml})\end{array}$ \\
\hline TMW 1.392 & $6.2 \times 10^{9} \pm 0.7 \times 10^{9}$ & $30.6 \pm 6.5$ \\
TMW 1.907 & $9.7 \times 10^{7} \pm 1.8 \times 10^{7}$ & $82.2 \pm 24.8$ \\
TMW 1.1150 & $1.2 \times 10^{10} \pm 0.2 \times 10^{10}$ & $60.0 \pm 12.4$ \\
TMW 1.2138 & $1.2 \times 10^{8} \pm 0.2 \times 10^{8}$ & $72.7 \pm 8.2$ \\
\hline
\end{tabular}

Table 2 Relation between lactate to acetate (FQ) and lactate to ethanol after $24 \mathrm{~h}$ of sourdough fermentation for sourdoughs of the $F$. sanfranciscensis strains TMW 1.392, TMW 1.907, TMW 1.1150 and TMW 1.2138 in combination without yeast (-yeasts), with K. humilis TMW 3.1034 and S. cerevisiae TMW 3.1064

\begin{tabular}{llll}
\hline & & $\begin{array}{l}\text { FQ (lactate/ } \\
\text { acetate) }\end{array}$ & Lactate/ethanol \\
\hline TMW 1.392 & -Yeast & 3.79 & 1.03 \\
& + K. humilis & 2.96 & 0.67 \\
& + S. cerevisiae & 2.17 & 0.45 \\
TMW 1.907 & -Yeast & 3.25 & 0.99 \\
& + K. humilis & 2.78 & 0.89 \\
& + S. cerevisiae & 2.26 & 0.56 \\
TMW 1.1150 & - Yeast & 3.71 & 1.02 \\
& + K. humilis & 3.17 & 0.96 \\
& + S. cerevisiae & 2.58 & 0.64 \\
TMW 1.2138 & - Yeast & 2.32 & 1.23 \\
& + K. humilis & 2.11 & 0.60 \\
& + S. cerevisiae & 2.23 & 0.35 \\
\hline
\end{tabular}

different, respectively. In TMW 1.907 only -yeast was different from the sourdoughs with yeast inoculation. The production of lactate was significant in the $F$. sanfranciscensis strains TMW 1.1150 and TMW 1.907 when inoculated with K. humilis TMW 3.1034.

\section{Discussion}

In former studies, the strain-dependent differences in the competitiveness in rye sourdough were investigated [23]. These competitive trials were also performed in the presence of the yeasts K. humilis TMW 3.1034 ( $+K$. humilis) or S. cerevisiae TMW 3.1064 ( + S. cerevisiae), or without any yeasts (-yeast). It was possible to categorize the eight tested $F$. sanfranciscensis strains into three different groups. F. sanfranciscensis TMW 1.1150 belonged to the group of dominating strains independently of yeast presence/absence. F. sanfranciscensis TMW 1.392 and TMW 1.907 belonged to the group for which the strain dominance was dependent on the presence/absence of yeast. F. sanfranciscensis TMW 1.907 prefers the presence of the yeast $S$. cerevisiae TMW 3.1064 whereas $F$. sanfranciscensis TMW 1.392 performed best with no added yeast in the fermentation. $F$. sanfranciscensis TMW 1.2138 belonged to the group of strains, which were not dominant in the sourdough competition independently of the presence or absence of yeasts [23]. In this study, the reasons for the different behavior in strain dominance were investigated along metabolic analyses. The metabolic turnover of $F$. sanfranciscensis strains was determined along the consumption of different carbohydrate sources and the concomitant production of acids and alcohols. The metabolite formation was normalized for comparison along the cell dry mass of the respective strain. This appeared as a better means compared to cell counts, because the cell size between strains varied significantly. This circumstance is apparently neglected in previous studies on the metabolism of $F$. sanfranciscensis strains but is considered as important. The metabolite analysis upon incubation with ribose or malate did not reveal any metabolic activity in any of the $F$. sanfranciscensis strains. The $F$. sanfranciscensis strain TMW 1.1150 was the most efficient per g/cell mass in maltose fermentation compared to the other four strains. This maltose fermentation or turnover within $6 \mathrm{~h}$ was neither influenced positively nor negatively by external electron acceptors. These results show that due to its rapid maltose fermentation this strain develops a rapid growth. Furthermore, the small cell structure is an advantage as cells with a higher surface to volume ratio are more effective [36]. In addition, this strain was also the most efficient in glucose fermentation, more efficient than any other strain (Fig. 2). Maltose and glucose are the main sugars in sourdough fermentation, as both are constantly produced by flour amylases [37]. A rapid fermentation of these sugars especially at the beginning of the fermentation leads to a growth benefit over the other strains. These other strains are not as fast as F. sanfranciscensis TMW 1.1150. This turnover can explain why $F$. sanfranciscensis TMW 1.1150 was dominant in all fermentations independently of the yeast co-inoculation. The addition of external electron acceptors did not influence the consumption of maltose, although an inoculation of yeasts in the sourdough increased its production of lactate, acetate and ethanol. In combination with K. humilis TMW 3.1034 the increase was more than with S. cerevisiae TMW 3.1064. Hence, there should be more factors above those ones determined in our study, which affect $F$. sanfranciscensis in the presence of yeasts. Indeed, stimulatory effects have been reported of nitrogen overflow, carbon dioxide or growth factors produced by $S$. cerevisiae on the survival of LAB in microbial communities in other LAB/yeast combinations $[10,38]$. 
F. sanfranciscensis TMW 1.2138 (Ls12) was the weakest in the competition studies. It was unable to compete against the other strains in any combination [23]. Although, its isolation from a sourdough shows that $F$. sanfranciscensis TMW 1.2138 can compete in this environment. As it is a strain from a wheat sourdough, it possibly would better persist in other sourdough types against other strains of $F$. sanfranciscensis or other LAB. However, in competitive studies in wheat flour of Siragusa et al. [39], this strain was also outcompeted by the autochthones wheat microbiota. In our studies, it was the slowest in the consumption of maltose, as after $6 \mathrm{~h}$ of fermentation time only $2.2 \mathrm{mM}$ of maltose were fermented (Fig. 2). Moreover, it was not able to ferment glucose, fructose or sucrose, which was also shown in previous studies [8]. A slow maltose fermentation and incapability of glucose fermentation explain why it is the weakest of the strains in sourdough fermentation. Furthermore, in the sourdough with $F$. sanfranciscensis TMW 1.2138, the presence or absence of the yeast did not alter the FQ as well as the production of the bacterial metabolites significantly. This result implies that the metabolic products of the yeasts do not affect the acetate level of $F$. sanfranciscensis TMW 1.2138 considerably (Table 2 ). The same observations were made before, as common electron acceptors like fructose and citrate did not alter the maltose fermentation of $F$. sanfranciscensis TMW 1.2138 significantly (Fig. 2) [8]. Moreover, only oxygen had a positive effect on maltose fermentation in the media fermentation. Though in combination with yeast in sourdoughs, the oxygen was consumed by the yeast's respiration [40]. In conclusion, these investigations explain why $F$. sanfranciscensis TMW 1.2138 belonged to the nondominant strains in competitiveness trials.

The $F$. sanfranciscensis strains TMW 1.392 and TMW 1.907 were influenced either negatively or positively by the presence of the yeasts K. humilis TMW 3.1034 and S. cerevisiae TMW 3.1064. F. sanfranciscensis TMW 1.392 preferred the absence of the yeasts although it is often found in combination with $K$. humilis [41]. This observation can be explained by the metabolic versatility of $F$. sanfranciscensis TMW 1.392. Notwithstanding, that its maltose turnover was lower for every condition than the turnover of maltose by $F$. sanfranciscensis TMW 1.1150, it was able to alter the fermentation the most (Fig. 2). The turnover of maltose by $F$. sanfranciscensis TMW 1.392 increased the most with electron acceptors like fructose and oxygen. Furthermore, it was able to use fructose and sucrose also for its metabolism and a clear turnover was detectable. When yeasts are present in the sourdough fermentation, the advantage of sucrose fermentation by $F$. sanfranciscensis TMW 1.392 is neglectable as sucrose is directly cleaved by the yeasts invertase [42, 43]. Moreover, the production of acetate and thereby the recycling of NAD and the extraction of an extra ATP is increased significantly yeast-dependent with $S$. cerevisiae
TMW 3.1064, more than with K. humilis TMW 3.1034 [37] (Table 2). Still, it appears that the advantage of an extra ATP through the acetate formation does not compensate for the lack of sucrose in sourdoughs with yeasts. Therefore, it can be explained why $F$. sanfranciscensis TMW 1.392 is not able to dominate in sourdoughs with yeasts together with $F$. sanfranciscensis TMW 1.1150 but without the yeasts [23].

Regarding F. sanfranciscensis TMW 1.907 it is difficult to explain why this strain is only dominant in combination with S. cerevisiae TMW 3.1046. In general, its turnover of maltose and glucose was better than in the weakest strain F. sanfranciscensis TMW 1.2138. It should be considered that their cell size and cell dry weight are similar (Table 1). Furthermore, its maltose turnover was increased by the presence of oxygen but not by fructose and citrate (Fig. 2). The production of the bacterial metabolites like lactate, acetate and mannitol was increased significantly in combination with yeasts. This effect can imply a stimulatory effect on the metabolism of $F$. sanfranciscensis TMW 1.907 by $S$. cerevisiae as well as $K$. humilis [10]. The higher production of ethanol in the sourdough fermentation is explained clearly by the alcoholic fermentation of the yeasts. An increase of ethanol formation is in sourdoughs with $F$. sanfranciscensis TMW 1.1150 and TMW 1.907 not as high as in sourdoughs with the other two strains (Fig. 4), although the ethanol concentrations are too low for inhibition of these species [7].

Taken together, the response of $F$. sanfranciscensis to the presence of yeasts is a strain- or group-specific trait. Generally, K. humilis revealed itself as a co-existing, i.e. commensal partner, which apparently neither elicit metabolic stress nor stimulation to $F$. sanfranciscensis, while the $S$. cerevisiae sourdough isolate rather showed competitive characteristics [23]. F. sanfranciscensis is in general nutrient competition with the $S$. cerevisiae, namely for maltose and sucrose, while the maltose-negative $K$. humilis prefers glucose [44]. Also, general mechanisms of redox-balance, e. g. thiol-metabolism, likely differ between yeast genera, and between $F$. sanfranciscensis strains, and may contribute to the strain-specific behavior observed [13, 14]. Depending on the $F$. sanfranciscensis partner the concomitant stress can therefore impose a negative effect on its competitiveness and metabolism in sourdoughs but can also be stimulatory. In this study, only F. sanfranciscensis TMW 1.907 was influenced positively by the presence of yeasts. In other combinations, the positive effects of the yeast interactions with $F$. sanfranciscensis are limited or absent, and the negative effects dominate, namely in combination with $S$. cerevisiae. Furthermore, the cell count of yeasts was decreased in combination with $F$. sanfranciscensis [23, 29], suggesting that these LAB also impose stress on the yeasts. This study, therefore, suggests that interactions of $F$. sanfranciscensis and the yeasts $S$. cerevisiae and $K$. humilis are competitive or commensal, respectively. 
Supplementary Information The online version contains supplementary material available at https://doi.org/10.1007/s00217-021-03722-0.

Funding Open Access funding enabled and organized by Projekt DEAL. Part of this work was supported by the German Ministry of Food and Agriculture (BMEL) in project 28-1-A1.039-16.

\section{Declarations}

Conflict of interest The authors declare that they have no conflict of interest.

Compliance with ethics requirements This article does not contain any studies with human or animal subjects.

Open Access This article is licensed under a Creative Commons Attribution 4.0 International License, which permits use, sharing, adaptation, distribution and reproduction in any medium or format, as long as you give appropriate credit to the original author(s) and the source, provide a link to the Creative Commons licence, and indicate if changes were made. The images or other third party material in this article are included in the article's Creative Commons licence, unless indicated otherwise in a credit line to the material. If material is not included in the article's Creative Commons licence and your intended use is not permitted by statutory regulation or exceeds the permitted use, you will need to obtain permission directly from the copyright holder. To view a copy of this licence, visit http://creativecommons.org/licenses/by/4.0/.

\section{References}

1. De Vuyst L, Neysens P (2005) The sourdough microflora: biodiversity and metabolic interactions. Trends Food Sci Technol $16(1-3): 43-56$

2. Van Kerrebroeck S, Maes D, De Vuyst L (2017) Sourdoughs as a function of their species diversity and process conditions, a metaanalysis. Trends Food Sci Technol 68:152-159. https://doi.org/10. 1016/j.tifs.2017.08.016

3. Yang H, Liu T, Zhang G, Chen J, Gu J, Yuan L, He G (2017) Genotyping of Lactobacillus sanfranciscensis isolates from Chinese traditional sourdoughs by multilocus sequence typing and multiplex RAPD-PCR. Int J Food Microbiol 258:50-57. https:// doi.org/10.1016/j.ijfoodmicro.2017.07.013

4. Zheng J, Wittouck S, Salvetti E, Franz CMAP, Harris HMB, Mattarelli P, O'Toole PW, Pot B, Vandamme P, Walter J, Watanabe K, Wuyts S, Felis GE, Gänzle MG, Lebeer S (2020) A taxonomic note on the genus Lactobacillus: description of 23 novel genera, emended description of the genus Lactobacillus Beijerinck 1901, and union of Lactobacillaceae and Leuconostocaceae. Int J Syst Evol Microbiol 70(4):2782-2858. https://doi.org/10.1099/ijsem.0. 004107

5. Brandt MJ, Gänzle M (2006) Handbuch Sauerteig. Behr's Verlag DE

6. Brandt MJ (2019) Industrial production of sourdoughs for the baking branch-an overview. Int J Food Microbiol 302:3-7. https:// doi.org/10.1016/j.ijfoodmicro.2018.09.008

7. Gänzle MG, Ehmann M, Hammes WP (1998) Modeling of growth of Lactobacillus sanfranciscensis and Candida milleri in response to process parameters of sourdough fermentation. Appl Environ Microbiol 64(7):2616-2623
8. Rogalski E, Ehrmann MA, Vogel RF (2020) Intraspecies diversity and genome-phenotype-associations in Fructilactobacillus sanfranciscensis. Microbiol Res. https://doi.org/10.1016/j.micres. 2020.126625

9. Zhang G, Tu J, Sadiq FA, Zhang W, Wang W (2019) Prevalence, genetic diversity, and technological functions of the Lactobacillus sanfranciscensis in sourdough: a review. Compr Rev Food Sci Food Saf 18(4):1209-1226

10. Sieuwerts S, Bron PA, Smid EJ (2018) Mutually stimulating interactions between lactic acid bacteria and Saccharomyces cerevisiae in sourdough fermentation. LWT 90:201-206. https://doi.org/10.1016/j.lwt.2017.12.022

11. Palla M, Blandino M, Grassi A, Giordano D, Sgherri C, Quartacci MF, Reyneri A, Agnolucci M, Giovannetti M (2020) Characterization and selection of functional yeast strains during sourdough fermentation of different cereal wholegrain flours. Sci Rep 10(1):12856. https://doi.org/10.1038/ s41598-020-69774-6

12. De Vuyst L, Harth H, Van Kerrebroeck S, Leroy F (2016) Yeast diversity of sourdoughs and associated metabolic properties and functionalities. Int J Food Microbiol 239:26-34. https://doi.org/ 10.1016/j.ijfoodmicro.2016.07.018

13. Jänsch A, Korakli M, Vogel RF, Gänzle MG (2007) Glutathione reductase from Lactobacillus sanfranciscensis DSM20451T: contribution to oxygen tolerance and thiol exchange reactions in wheat sourdoughs. Appl Environ Microbiol 73(14):4469-4476. https://doi.org/10.1128/aem.02322-06

14. Stetina M, Behr J, Vogel RF (2014) The transcriptional response of Lactobacillus sanfranciscensis DSM $20451^{\mathrm{T}}$ and its $t c y B$ mutant lacking a functional cystine transporter to diamide stress. Appl Environ Microbiol 80(14):4114-4125. https://doi.org/10. 1128/aem.00367-14

15. Gobbetti M, Corsetti A, Rossi J (1995) Maltose-fructose cofermentation by Lactobacillus brevis subsp. lindneri CB1 fructose-negative strain. Appl Microbiol Biotechnol 42(6):939-944. https://doi.org/10.1007/BF00191194

16. Corsetti A, Settanni L (2007) Lactobacilli in sourdough fermentation. Food Res Int 40(5):539-558. https://doi.org/10.1016/j.foodr es.2006.11.001

17. Foschino R, Arrigoni C, Mora D, Galli A (2001) Phenotypic and genotypic aspects of Lactobacillus sanfranciscensis strains isolated from sourdoughs in Italy. Food Microbiol 18(3):277-285

18. Korakli M, Vogel RF (2003) Purification and characterisation of mannitol dehydrogenase from Lactobacillus sanfranciscensis. FEMS Microbiol Lett 220(2):281-286

19. Kaditzky S, Seitter M, Hertel C, Vogel RF (2008) Performance of Lactobacillus sanfranciscensis TMW 1.392 and its levansucrase deletion mutant in wheat dough and comparison of their impact on bread quality. Eur Food Res Technol 227(2):433-442. https:// doi.org/10.1007/s00217-007-0738-1

20. Tieking M, Ehrmann MA, Vogel RF, Gänzle MG (2005) Molecular and functional characterization of a levansucrase from the sourdough isolate Lactobacillus sanfranciscensis TMW 1.392. Appl Microbiol Biotechnol 66(6):655-663. https://doi.org/10. 1007/s00253-004-1773-5

21. Stolz P, Vogel RF, Hammes WP (1995) Utilization of electron acceptors by Lactobacilli isolated from sourdough. Zeitschrift für Lebensmittel-Untersuchung und Forschung 201(4):402-410. https://doi.org/10.1007/bf01192742

22. Vogel RF, Ehrmann MA, Gänzle MG (2002) Development and potential of starter Lactobacilli resulting from exploration of the sourdough ecosystem. Antonie Van Leeuwenhoek 81(1):631-638. https://doi.org/10.1023/A:1020530227192 
23. Rogalski E, Ehrmann MA, Vogel RF (2020) Role of Kazachstania humilis and Saccharomyces cerevisiae in the strain-specific assertiveness of Fructilactobacillus sanfranciscensis strains in rye sourdough. Eur Food Res Technol. https://doi.org/10.1007/ s00217-020-03535-7

24. Hammes WP, Stolz P, Gänzle MG (1996) Metabolism of Lactobacilli in traditional sourdoughs. Adv Food Sci 18(5-6):176-184

25. Iosca G, Vero LD, Gullo M, Licciardello F, Quartieri A, Pulvirenti A (2020) Exploring the microbial community of traditional sourdoughs to select yeasts and lactic acid bacteria. In: Multidisciplinary digital publishing institute proceedings, vol 1, p 3

26. Ripari V, Gänzle MG, Berardi E (2016) Evolution of sourdough microbiota in spontaneous sourdoughs started with different plant materials. Int J Food Microbiol 232:35-42. https://doi.org/10. 1016/j.ijfoodmicro.2016.05.025

27. Gänzle MG, Zheng J (2019) Lifestyles of sourdough lactobacilli-do they matter for microbial ecology and bread quality? Int J Food Microbiol 302:15-23. https://doi.org/10.1016/j.ijfoo dmicro.2018.08.019

28. Kayikci O, Nielsen J (2015) Glucose repression in Saccharomyces cerevisiae. FEMS Yeast Res. https://doi.org/10.1093/femsyr/ fov068

29. Carbonetto B, Nidelet T, Guezenec S, Perez M, Segond D, Sicard D (2020) Interactions between Kazachstania humilis yeast species and lactic acid bacteria in sourdough. Microorganisms. https://doi. org/10.3390/microorganisms 8020240

30. Gänzle M, Ripari V (2016) Composition and function of sourdough microbiota: from ecological theory to bread quality. Int $\mathbf{J}$ Food Microbiol 239:19-25. https://doi.org/10.1016/j.ijfoodmicro. 2016.05.004

31. Kitahara M, Sakata S, Benno Y (2005) Biodiversity of Lactobacillus sanfranciscensis strains isolated from five sourdoughs. Lett Appl Microbiol 40(5):353-357. https://doi.org/10.1111/j.1472765X.2005.01678.x

32. Rogalski E, Vogel RF, Ehrmann MA (2020) Monitoring of Lactobacillus sanfranciscensis strains during wheat and rye sourdough fermentations by CRISPR locus length polymorphism PCR. Int J Food Microbiol 316:108475. https://doi.org/10.1016/j.ijfoodmicro.2019.108475

33. Rühmkorf C, Jungkunz S, Wagner M, Vogel RF (2012) Optimization of homoexopolysaccharide formation by lactobacilli in gluten-free sourdoughs. Food Microbiol 32(2):286-294. https:// doi.org/10.1016/j.fm.2012.07.002

34. Cabálková J, Žídková J, Přibyla L, Chmelík J (2004) Determination of carbohydrates in juices by capillary electrophoresis, highperformance liquid chromatography, and matrix-assisted laser desorption/ionization-time of flight-mass spectrometry. Electrophoresis 25(3):487-493. https://doi.org/10.1002/elps.200305672
35. Schmid J, Bechtner J, Vogel RF, Jakob F (2019) A systematic approach to study the $\mathrm{pH}$-dependent release, productivity and product specificity of dextransucrases. Microb Cell Fact 18(1):153. https://doi.org/10.1186/s12934-019-1208-8

36. Harris LK, Theriot JA (2018) Surface area to volume ratio: a natural variable for bacterial morphogenesis. Trends Microbiol 26(10):815-832. https://doi.org/10.1016/j.tim.2018.04.008

37. Gänzle MG, Vermeulen N, Vogel RF (2007) Carbohydrate, peptide and lipid metabolism of lactic acid bacteria in sourdough. Food Microbiol 24(2):128-138. https://doi.org/10.1016/j.fm.2006. 07.006

38. Ponomarova O, Gabrielli N, Sévin DC, Mülleder M, Zirngibl K, Bulyha K, Andrejev S, Kafkia E, Typas A, Sauer U, Ralser M, Patil KR (2017) Yeast creates a niche for symbiotic lactic acid bacteria through nitrogen overflow. Cell Syst 5(4):345-357.e346. https://doi.org/10.1016/j.cels.2017.09.002

39. Siragusa S, Di Cagno R, Ercolini D, Minervini F, Gobbetti M, De Angelis M (2009) Taxonomic structure and monitoring of the dominant population of lactic acid bacteria during wheat flour sourdough type I propagation using Lactobacillus sanfranciscensis starters. Appl Environ Microbiol 75(4):1099-1109. https://doi. org/10.1128/aem.01524-08

40. Malakar S, Paul SK, Jolvis Pou KR (2020) 1-biotechnological interventions in beverage production. In: Grumezescu AM, Holban AM (eds) Biotechnological progress and beverage consumption. Academic Press, pp 1-37. https://doi.org/10.1016/B978-012-816678-9.00001-1

41. Venturi M, Guerrini S, Vincenzini M (2012) Stable and non-competitive association of Saccharomyces cerevisiae, Candida milleri and Lactobacillus sanfranciscensis during manufacture of two traditional sourdough baked goods. Food Microbiol 31(1):107-115

42. Fujimoto A, Ito K, Itou M, Narushima N, Ito T, Yamamoto A, Hirayama S, Furukawa S, Morinaga Y, Miyamoto T (2018) Microbial behavior and changes in food constituents during fermentation of Japanese sourdoughs with different rye and wheat starting materials. J Biosci Bioeng 125(1):97-104. https://doi.org/10. 1016/j.jbiosc.2017.08.009

43. Loponen J, Gänzle MG (2018) Use of sourdough in low FODMAP baking. Foods 7(7):96

44. Sidari R, Martorana A, Zappia C, Mincione A, Giuffrè AM (2020) Persistence and effect of a multistrain starter culture on antioxidant and rheological properties of novel wheat sourdoughs and bread. Foods 9(9):1258

Publisher's Note Springer Nature remains neutral with regard to jurisdictional claims in published maps and institutional affiliations. 